\title{
Left ventricular function during balloon dilatation of the aortic valve in elderly patients: a blind study of echocardiograms
}

\author{
Henrik Egeblad, Alf Wennevold
}

\begin{abstract}
Subcostal echocardiography of the left ventricle was continuously recorded during balloon dilatation of the aortic valve in ten consecutive elderly patients. Left ventricular wall motion decreased gradually during a minute of maintained complete balloon inflation. Blind analysis of $M$ mode echocardiograms showed a significant reduction in fractional shortening. Deflation of the balloon resulted in global left ventricular hyperkinesia. There was a transient overshoot in fractional shortening, wall thickness, and blood pressure approximately $40 \mathrm{~s}$ after deflation of the balloon. The myocardial reaction was similar to that known to occur regionally with brief coronary artery obstruction, whereas the timing of the events seemed to be dissociated from the timing of changes in left ventricular load. Thus it is likely that the left ventricular response during valve dilatation reflects not only changes in load but also an ischaemia-reperfusion effect on the myocardium. The systolic thickening of the hypertrophied interventricular septum was slightly reduced after balloon dilatation. This finding might be a result of repeated episodes of ischaemia and reperfusion. However, the potential for myocardial injury seemed to be unimportant clinically.
\end{abstract}

Balloon dilatation is increasingly used to treat elderly patients with aortic valve stenosis. ${ }^{1-3}$ The cardiovascular response to the repeated inflations and deflations of the balloon is complex. Transient circulatory obstructions cause considerable changes in left ventricular load. In addition, ischaemia and reperfusion may have a direct and potentially injurious effect on the myocardium. Brief obstruction of a coronary artery is known to result in characteristic abnormalities in regional wall motion. ${ }^{4-6}$ The aim of this study was to examine whether there are similar global myocardial reactions in the left ventricle during balloon dilatation of the aortic valve.

\section{Patients and methods}

We studied 10 consecutive patients (four men, six women; median age 76 years (range 72-87 years)) who underwent balloon dilatation of the aortic valve. All had clinical, electrocardiographic, radiological, and echocardiographic findings indicating severe stenosis of the aortic valve. Three of the patients had coexistent aortic valve incompetence that was considered to be haemodynamically insignificant on the basis of physical examination. None of the patients had primary mitral valve disease. Open heart operation for aortic valve stenosis was considered inappropriate because of the patient's advanced age or coexistent disease.

\section{INVASIVE PROCEDURE}

A $7 \mathrm{~F}$ pigtail catheter was introduced percutaneously into the femoral artery and advanced to the ascending aorta and on to the left ventricle with the aid of a guide wire. The peak gradient over the aortic valve was calculated as the difference between peak left ventricular systolic pressure and peak systolic pressure in the ascending aorta. The guide wire was used to replace the pigtail catheter by a Meditech $15 \mathrm{~mm}(3-4 \mathrm{~cm}$ ) balloon catheter. The aortic valve was dilated with the centre of the balloon at the level of the aortic valve, which was apparent from the calcifications seen by fluoroscopy. The balloon was inflated with a mixture of saline and Urografin $76 \%$. Inflation was maintained at constant maximum manual pressure for $60 \mathrm{~s}^{1}$ unless the patient showed signs of insufficient cerebral perfusion. On fewer than 10 occasions was it necessary to deflate the balloon after 40 or $50 \mathrm{~s}$ because of vertigo, obvious dyspnoea, or when the patients speech became slowed. None of the patients lost consciousness or developed chest pain during the procedure.

Repeated dilatations were performed every 1-2 min, or more if the balloon catheter was replaced by a balloon of a larger size. Where possible dilatations were routinely carried out with balloons of increasing sizes from 15 to 18 or $20 \mathrm{~mm}$ in diameter. The procedure was stopped after 3-5 dilatations with the largest balloon. A median of eight (range 3-11) inflations of a balloon in the aortic ostium were carried out in the 10 patients.

Blood pressure was continuously recorded through an arterial sheath. Cardiac output was not measured. Because these were elderly patients we did not perform ventriculography, aortography, or coronary arteriography.

NON-INVASIVE PROCEDURE

Cross sectional and $M$ mode echocardiograms from the parasternal, apical, and subcostal positions were recorded on video tape before and after balloon dilatation. During dilatation 

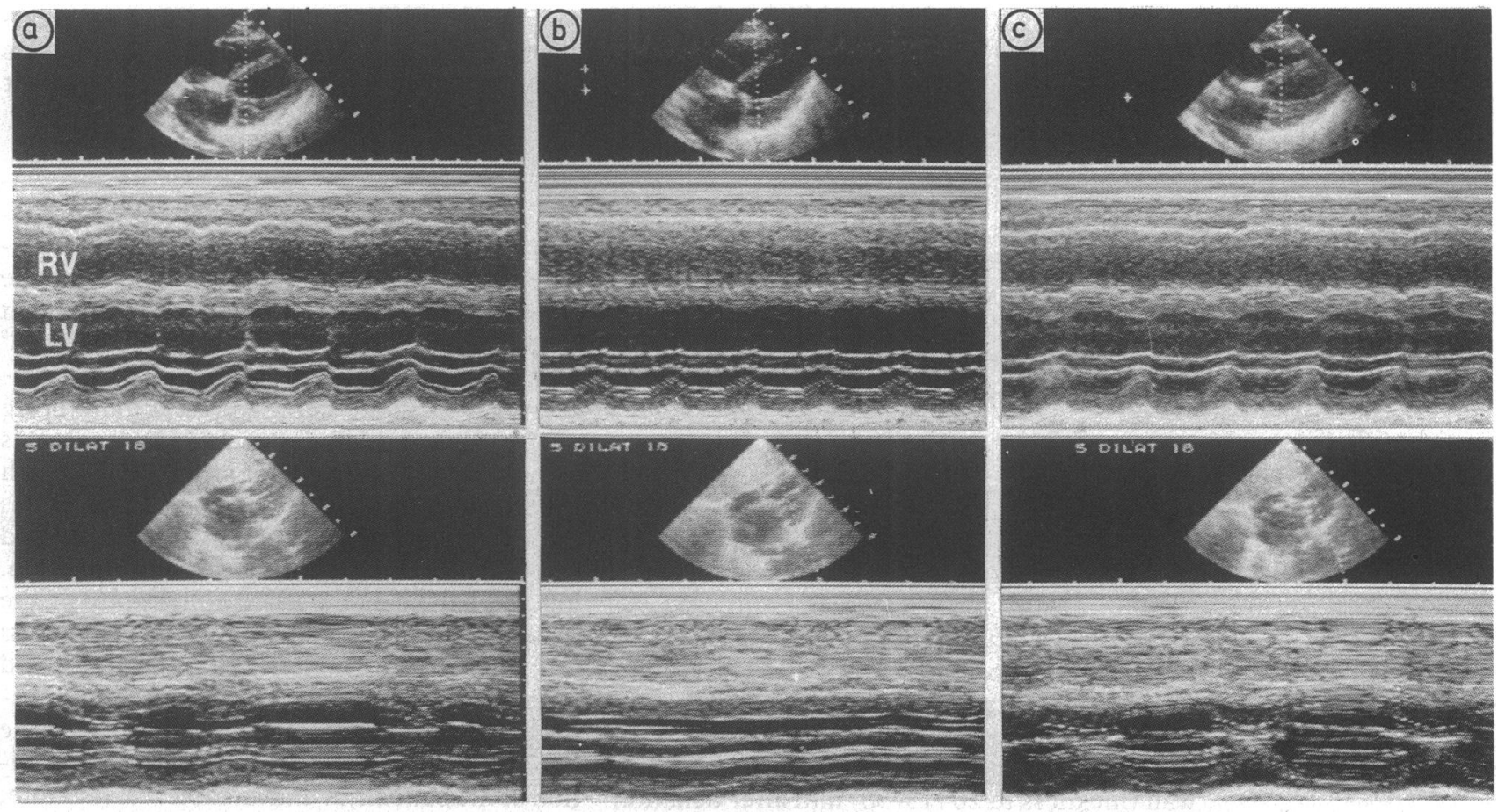

Figure $1 M$ mode echocardiograms guided by cross sectional subcostal four chamber view in two patients undergoing balloon dilatation of the aortic valve. Echocardiograms obtained immediately before balloon obstruction of the aortic valve (a); at the end of 1 min obstruction (b); and 40 s after deflation of the balloon (c). In one patient (above) the septum between right ( $R V$ ) and left ventricle (LV) and the lateral wall (below LV) are clearly defined. The echocardiograms of the second patient (below) show even more pronounced hypokinesia during balloon obstruction and hyperkinesia during reperfusion, but the definition of the septum is more vague because of right ventricular obliteration in the projection selected.

simultaneous cross sectional and $M$ mode echocardiography were performed from the subcostal position. An $M$ mode diameter through the left ventricle at the level of the chordae of the mitral valve was selected on the cross sectional four chamber view. Echocardiograms were continuously recorded on video tape before each inflation of the balloon, during maintained complete balloon inflation, and during and after deflation of the balloon $(25 \mathrm{~mm} / \mathrm{s}$ or $50 \mathrm{~mm} / \mathrm{s})$. Polaroid photographs were taken from the video tapes including recordings obtained before and after dilatation and immediately before and at the end of each balloon inflation (fig 1). In addition, Polaroid recordings were obtained from the video tape after each deflation of the balloon when left ventricular wall motion was re-established and seemed from the cross sectional image to be at its maximum (fig 1). Exaggerated respiratory movements occasionally distorted the echocardiogram. In these patients photographs were taken of the video tapes as close to the scheduled time as possible.

The identity of the patient and the timing of the recording were marked on the reverse of each photo. They were later shuffled and analysed in random order without knowledge of at what stage of the procedure they were obtained. We measured left ventricular diameter at end diastole (Dd) and end systole (Ds) and septal (ST) and lateral wall (LW) thickness. Left ventricular fractional shortening $(\mathrm{FS}=((\mathrm{Dd}-\mathrm{Ds}) / \mathrm{Dd}) \quad \times \quad 100 \%) \quad$ and thickening of the septum and lateral wall from diastole to systole were calculated. All measurements were obtained as mean values of 3-5 cycles.

The heart rate and number of runs of three or more ventricular extrasystoles in relation to inflation of the balloon were calculated from the continuously recorded electrocardiogram.

We used the Wilcoxon test for statistical analysis and a $p$ value below 5\% was regarded as significant.

\section{Results}

In the subcostal four chamber view the left ventricle appeared gradually to develop diffuse hypokinesia or akinesia over the $60 \mathrm{~s}$ period of maintained balloon inflation, with subsequent gradual development of global hyperkinesia after deflation of the balloon. These changes accorded with the measurements taken from the $M$ mode recordings (figs 1 and 2). The median values and ranges shown in fig 2 include all values obtained immediately before inflation of the balloon, $60(15-60) \mathrm{s}$ after complete inflation, and $40(10-60) \mathrm{s}$ after the deflations. The most pronounced echocardiographic changes were associated with fractional shortening. There was a significant reduction from $30(12-72) \%$ before obstruction to 26 (9$56) \%$ during obstruction and a significant increase to $43(15-79) \%$ after deflation. A reduction in systolic thickening of the lateral wall from $6(1-13) \mathrm{mm}$ before obstruction to 4 (0-10) $\mathrm{mm}$ during obstruction was statistically significant; systolic thickening of the septum was reduced from $4(0-18) \mathrm{mm}$ to $3(0-14) \mathrm{mm}$ $(p>0.05)$. After deflation systolic thickening of both the lateral wall and septum increased to $7(0-15) \mathrm{mm}$ and $7(0-18) \mathrm{mm}$ respectively ( $p<0.05$ for both).

The diastolic thickness of the septum and lateral wall did not change significantly during obstruction (before obstruction $16(8-30) \mathrm{mm}$ 


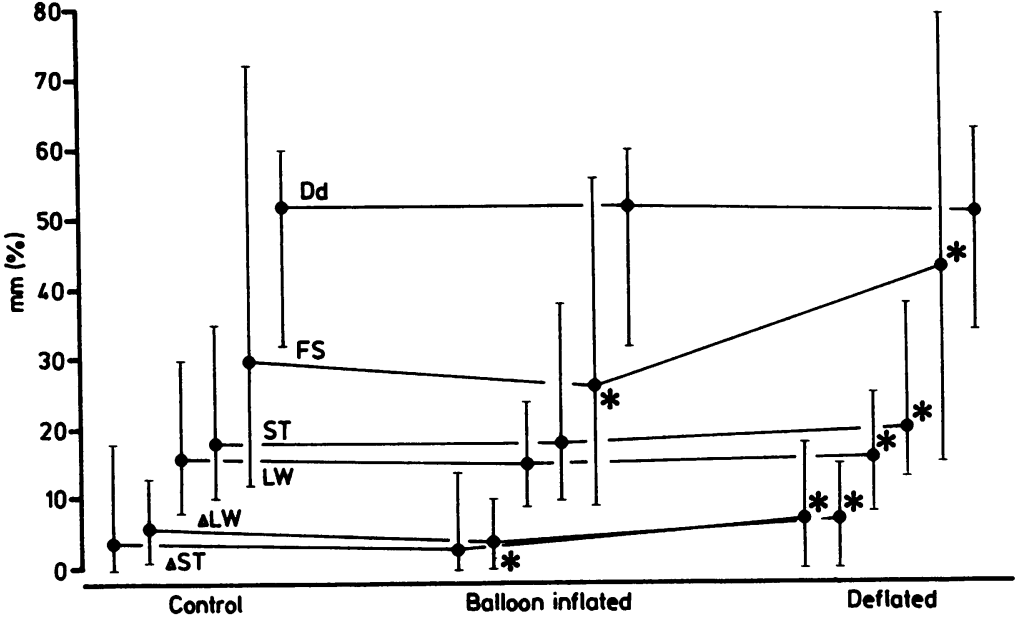

Figure 2 Variation in left ventricular variables measured by subcostal echocardiography during balloon dilatation of the aortic valve. Median values and range are shown in 10 patients who had 8 (3-11) balloon inflations performed. Values measured after 60 (15-60) s obstruction of the aortic ostium are compared with preobstructive values obtained immediately before balloon inflation and with values measured $40(10-60)$ after deflation of the balloon. See footnotes to tables for abbreviations. ${ }^{\star} p<0.05$ compared with preceding value (rank sum test for paired data) for lateral wall thickness and $18(10-35) \mathrm{mm}$ for septal thickness compared with 15 (9-24) mm for lateral wall and $18(10-38) \mathrm{mm}$ for septal thickness during obstruction). Lateral wall thickness increased to $16(8-25) \mathrm{mm}$ and septal wall thickness to 20 (13-38) $\mathrm{mm}$ after deflation of the balloon ( $p<0.05$ for both). Left ventricular diameter at end diastole did not change significantly, either from before obstruction $(52(32-60) \mathrm{mm})$ to the value measured after $60 \mathrm{~s}$ of obstruction $(52(32-60) \mathrm{mm})$ or that measured $40 \mathrm{~s}$ after deflation of the balloon ( 51 $(34-64) \mathrm{mm}$ ).

Heart rate increased from $92(64-135)$ beats/ min before to $100(65-122)$ beats/min during obstruction and slowed to $95(64-135)$ beats/ min $40(10-60)$ s after deflation, but these variations were not statistically significant. Blood pressure fell from $105(68-175) / 50$ (30-70) $\mathrm{mm} \mathrm{Hg}$ to 68 (24-95)/38 (1755) $\mathrm{mm} \mathrm{Hg}$ from baseline to balloon obstruction ( $p<0.05$ for systolic and diastolic blood pressure) and increased to 135 (65-195)/50 $(30-100) \mathrm{mm} \mathrm{Hg} 40(30-90) \mathrm{s}$ after deflation of the balloon ( $p<0.05$ for both). Only one episode of three or more runs of ventricular extrasystoles was recorded before balloon inflation whereas runs of ventricular extrasystoles were seen in $43 \%$ of the periods after inflation of the balloon (5 (0-12) s after inflation) and in $30 \%$ of the periods after deflation $(45(18-80) \mathrm{s}$ after deflation).

Values recorded for the first cycle of balloon inflation and deflation and for the last cycle were not significantly different (table 1 ).

Data recorded before the procedure were compared with those obtained after the treatment (table 2). Aortic valve opening was significantly increased and the valve gradient and septal thickening decreased significantly.

\section{Discussion}

Previous studies showed that echocardiography may be a useful aid to guiding the balloon catheter and for evaluation of the result of balloon dilatation of the aortic valve. ${ }^{7-9}$ Transoesophageal echocardiography in particular may facilitate stable and clear monitoring of the valve and left ventricle. ${ }^{9}$ However, this technique requires general anaesthesia, which is best avoided in elderly patients and impedes the evaluation of the tolerance of decreased cerebral perfusion during balloon inflation. Parasternal M mode echocardiography of the left ventricle may be difficult to perform unless the patient is in the left lateral position, and interference between the ultrasound transducer, the hand of the examiner, and the fluoroscope, which is used to control the position of the balloon, is unavoidable. ${ }^{10}$ Therefore, in this study we monitored left ventricular wall motion from the subcostal view. Continuous guidance by cross sectional echocardiography was helpful for maintenance of the direction of the $M$ mode echocardiogram although optimum definition of the cardiac walls could not be obtained in all patients (fig 1). However, the reliability of the variations actually detected was endorsed by blind analysis of the echocardiograms and by previously reported experimental and clinical data.

Left ventricular hypokinesia during balloon obstruction-in this study recorded by echocardiography (figs 1 and 2)-has recently been confirmed by angiography. ${ }^{11}$ Hypokinesia may occur because of the considerable increase in left ventricular afterload, but may also be a result of ischaemia. Reduction of cardiac output and aortic pressure owing to the balloon inflation together with possible obstruction of the coronary artery ostia may diminish myocardial oxygen supply. The ensuing reduction of the pump function might complete a vicious circle. The hypertrophy of the left ventricular myocardium (table 2) and possible coronary artery disease in some of the patients could further increase the sensitivity to reduced blood pressure, with the subendocardium being particularly jeopardised. ${ }^{12}$ Coronary arteriography was not performed in our

Table 1 Data (median (range)) measured during balloon dilatation of the aortic valve

\begin{tabular}{|c|c|c|c|c|c|c|}
\hline & \multicolumn{2}{|c|}{ Preobstructive control } & \multicolumn{2}{|c|}{ Balloon inflated } & \multicolumn{2}{|c|}{ Balloon deflated } \\
\hline & First value & Last value & First value & Last value & First value & Last value \\
\hline $\begin{array}{l}\text { Dd (mm) } \\
\text { FS (\%) } \\
\text { ST (mm) } \\
\text { LW (mm) } \\
\triangle S T(m m) \\
\triangle \mathbf{L W}(\mathrm{mm}) \\
\text { Systolic BP (mm Hg) } \\
\text { Diastolic BP (mm Hg) } \\
\text { HR (beats/min) }\end{array}$ & $\begin{array}{c}52(40-59) \\
28(19-58) \\
17(13-32) \\
13(11-18) \\
4(1-9) \\
6(4-8) \\
125(80-158) \\
50(40-62) \\
84(70-107)\end{array}$ & $\begin{array}{c}48(39-60) \\
27(12-79) \\
17(13-35) \\
13(11-22) \\
3(0-12) \\
6(2-9) \\
145(68-160) \\
50(30-64) \\
98(70-112)\end{array}$ & $\begin{array}{l}49(35-56) \\
25(13-43) \\
18(12-31) \\
14(9-18) \\
4(2-7) \\
4(3-10) \\
75(52-95) \\
40(35-55) \\
90(78-110)\end{array}$ & $\begin{array}{l}51(34-59) \\
18(14-56) \\
18(13-28) \\
13(10-24) \\
3(0-9) \\
3(1-7) \\
50(35-65) \\
37(25-50) \\
90(68-122)\end{array}$ & $\begin{array}{c}50(40-59) \\
47(17-57) \\
18(14-28) \\
16(10-20) \\
5(0-12) \\
6(0-15) \\
140(65-165) \\
42(38-65) \\
90(82-115)\end{array}$ & $\begin{array}{c}48(34-57) \\
36(20-65) \\
18(15-34) \\
15(8-22) \\
5(1-12) \\
6(4-14) \\
135(74-168) \\
45(42-60) \\
99(68-118)\end{array}$ \\
\hline
\end{tabular}

Left ventricular characteristics by subcostal echocardiography, heart rate (HR), and blood pressure (BP) measured initially and late during the course of balloon dilatation of the aortic valve. Dd, left ventricular diastolic diameter; FS, left ventricular fractional shortening; LW, lateral wall thickness in diastole; $\triangle \mathrm{LW}$, systolic thickening of the lateral wall; ST, thickness of the interventricular septum in diastole, $\triangle S T$, systolic thickening of the interventricular septum. 
Table 2 Data (median (range)) measured before and after balloon dilatation of the aortic valve

\begin{tabular}{|c|c|c|}
\hline & Before & After \\
\hline $\begin{array}{l}\text { Time of examination } \\
\text { in relation to procedure } \\
\text { (days) }\end{array}$ & $3(1-7)$ & $4(1-11)$ \\
\hline $\begin{array}{l}\text { Parasterm } \\
\text { Aortic cusp separation } \\
\text { Dd (mm) } \\
\text { Ds (mm) } \\
\text { FS (\%) } \\
\text { ST (mm) } \\
\text { PW (mm) }\end{array}$ & $\begin{array}{c}\text { al echocardiography } \\
4(3-6) \\
52(39-62) \\
38(24-52) \\
32(17-40) \\
15(12-18) \\
12(8-17)\end{array}$ & $\begin{array}{l}6(3-9)^{\star} \\
58(37-65) \\
43(15-51) \\
29(14-59) \\
15(12-20) \\
13(10-15)\end{array}$ \\
\hline $\begin{array}{l} \\
\text { Dd (mm) } \\
\text { Ds (mm) } \\
\text { FS (\%) } \\
\text { ST (mm) } \\
\text { LW (mm) } \\
\triangle \text { ST (mm) } \\
\triangle \mathrm{LW}(\mathrm{mm}) \\
\left.\text { HR (beats } \times \mathrm{min}^{-1}\right) \\
\text { BP (mm Hg) }\end{array}$ & $\begin{array}{l}\text { echocardiography } \\
53(37-63) \\
38(18-52) \\
28(14-51) \\
17(13-34) \\
14(10-21) \\
5(0-9) \\
5(2-8) \\
85(65-107) \\
130(100-155) / \\
70(60-100)\end{array}$ & $\begin{array}{c}55(37-59) \\
39(18-48) \\
24(16-53) \\
17(14-37) \\
13(12-21) \\
3(0-7)^{\star} \\
5(2-12) \\
90(72-107) \\
130(100-165) / \\
80(60-90)\end{array}$ \\
\hline $\begin{array}{l}\text { Aortic valve gradient } \\
\text { immediately before and } \\
\text { after procedure }(\mathrm{mm} \mathrm{Hg})\end{array}$ & $80(30-125)$ & $30(0-55)^{\star}$ \\
\hline
\end{tabular}

Left ventricular characteristics, heart rate (HR), and blood pressure (BP) before and after balloon dilatation of the aortic pressure (BP) before and after balloon dilatation of the aortic
valve. Dd, left ventricular diastolic diameter; Ds, left valve. Dd, left ventricular diastolic diameter; Ds, left ventricular systolic diameter; FS, left ventricular fractional shortening; LW, lateral wall thickness in diastole; $\Delta L W$,
systolic thickening of lateral wall; $P W$, posterior wall thickness in diastole; ST, thickness of the interventricular septum in diastole; $\triangle \mathrm{ST}$, systolic thickening of the interventricular septum. ${ }^{*} \mathrm{p}<0.05$

patients, none of whom developed angina pectoris during balloon dilatation. However, transient ST-T depression was often seen on the monitor electrocardiogram. The ST-T depressions were not measured because electrocardiographic mapping would have interfered with the fluoroscopy. Furthermore, bundle branch block and treatment with digoxin may interfere with the measurement and interpretation of ST-T changes in many elderly patients with aortic stenosis.

Complete coronary artery obstruction by ligation leads to regional dyskinesia within a minute. ${ }^{413}$ Dyskinesia is often noticed in anterior wall infarction with $Q$ waves but rarely in non- $Q$ wave infarction and in pathoanatomical subendocardial infarction. ${ }^{14}{ }^{15}$ Most of our patients showed hypokinesia during balloon inflation but a few showed akinesia (fig 1). Dyskinesia did not occur. Left ventricular wall motion gradually became hypokinetic or akinetic within $60 \mathrm{~s}$ of complete inflation of the balloon. The left ventricular diameter at end diastole did not change significantly (fig 2). Thus the increase in left ventricular load remained constant while the balloon was kept inflated. Increased afterload doubtlessly contributed to the left ventricular response when the balloon was kept inflated. However, the timing and character of the wall motion abnormality were compatible with the concept of subendocardial ischaemia as a determinant of the development of hypokinesia and akinesia during the balloon obstruction. This hypothesis is supported by the recent demonstration by catheterisation of the coronary sinus of transient low flow ischaemia during balloon inflation..$^{16}$

The response of the left ventricle to deflation of the balloon included transient hyperkinesia of the left ventricular walls that reached a maximum $40 \mathrm{~s}$ after the start of deflation.
There was no significant change in the left ventricular diameter at end diastole. Deflation of the balloon led to a reduction of the left ventricular afterload. A decrease of peripheral vascular resistance is likely to occur at the same time. Cardiac output was not monitored in this study but a reduction in peripheral vascular resistance was recognised in studies of declamping after aortic surgery. ${ }^{17}$ The reduction in afterload resulting from the balloon deflation and the potential reduction of peripheral vascular resistance accord with the suggestion that there is left ventricular hyperkinesia after obstruction by the balloon. However, reperfusion of the myocardium may contribute significantly to the increased wall motion. A hyperkinetic wall motion response, which was attributed to sudden cellular calcium influx, was seen in experimental and clinical studies after the abolition of brief regional myocardial ischaemia. ${ }^{4513}$ In animal experiments ligation of the coronary artery for a minute resulted in maximum hyperkinesia after $40 \mathrm{~s}$ of reperfusion. ${ }^{13} \mathrm{~A}$ similar time course of hypokinesia and hyperkinesia was seen in variant angina. ${ }^{18}$ Furthermore, the significant increase in diastolic wall thickness after deflation of the balloon resembles the findings at coronary artery reperfusion in the experimental setting. ${ }^{13} 19$ The increase in wall thickness has been attributed to engorgement of the myocardial vascular bed during reactive hyperaemia. The gradual increase in left ventricular wall motion and systolic blood pressure during reperfusion, with an overshoot in both after $40 \mathrm{~s}$ and a simultaneous diastolic blood pressure value at the pre-obstructive level are likely to reflect myocardial reperfusion rather than a decrease of afterload. The reduction in afterload may be greatest immediately after the balloon deflation. Finally, the occurrence of arrhythmias after deflation of the balloon indicates a reperfusion effect on the myocardium. ${ }^{20}$

The variation in fractional shortening was further analysed to obtain a clearer idea of the time course of the myocardial response to inflation and deflation of the balloon (fig 3). To limit the number of measurements, the analysis was confined to two patients. Their $M$ mode video recordings of the left ventricle were stable and during all aortic valve dilatations the balloon was completely inflated for $60 \mathrm{~s}$. The number of balloon inflations in both these patients resembled the median number of dilatations (eight) in the study population. The two patients are not those shown in fig 1 . An additional 161 photographs were obtained from the two patients' video tapes immediately before the procedure and every $15 \mathrm{~s}$ during and after $60 \mathrm{~s}$ of maintained complete inflation of the balloon. Fractional shortening was measured without knowledge of the timing of the recordings. There was no significant reduction of fractional shortening until late during the $60 \mathrm{~s}$ period of maintained inflation (fig 3 ). Other data from our laboratory showed that the time needed for complete filling and emptying of the balloon was approximately 20 s. Figure 3 indicates that there was no reduction of fractional shortening during filling of the balloon 
Figure 3 Fractional shortening (median and range) recorded every $15 \mathrm{~s}$ in two patients $(1 \mathrm{M}, 1 \mathrm{~F})$ in whom seven and 10 balloon inflations and deflations were performed. $C$, value recorded immediately before the procedure. I, the $60 \mathrm{~s}$ period during which complete balloon inflation was maintained. Deflation started at D. In most case a new balloon inflation was carried out 60 s after $D$. Open asterisks, $p<0.05$ compared with control value; closed asterisks, $p<0.05$ compared with value $15 \mathrm{~s}$ before (rank sum test for paired data).

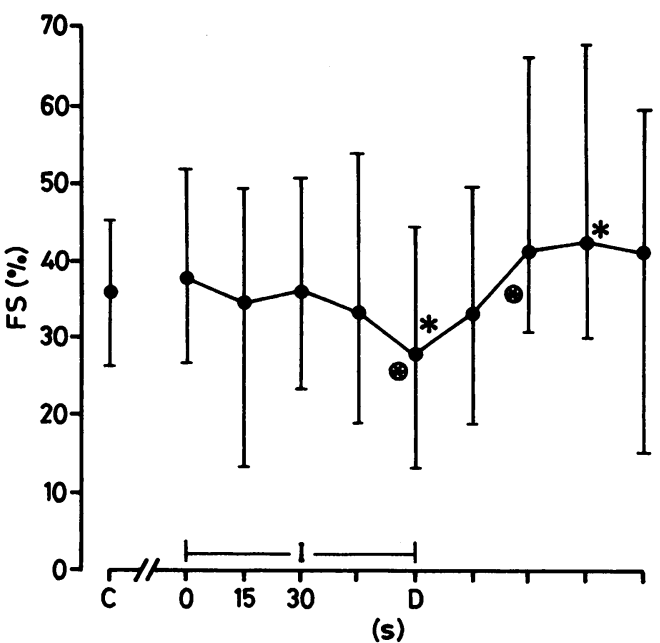

(before I in fig 3). Therefore, partial obstruction of the aorta during emptying of the balloon cannot be regarded as a likely explanation of the delay of maximum wall motion hyperkinesia (fig 3). Furthermore, the entire delay of 40-45 s after the start of deflation cannot be explained by the $20 \mathrm{~s}$ needed to deflate the balloon completely. However, the time course of fractional shortening during inflation and deflation of the balloon is similar to the time course of regional wall motion observed in experimental ischaemia and reperfusion. ${ }^{13}$

Recent studies have shown minor improvement of ejection fraction immediately, early, and late after balloon dilatation of the aortic valve. ${ }^{21}$ Fractional shortening did not change significantly after balloon dilatation in this study (table 2). This does not exclude an increase in overall systolic function because only the function of myocardial fibres at the base of the ventricle is reflected in fractional shortening.

Throughout the procedure there were insignificant reductions in fractional shortening and systolic septal thickening (table 1). However, the pre-obstructive values of fractional shortening and systolic septal and lateral wall thickening before the last balloon inflation may have been artificially increased by the postobstructive wall motion hyperkinesia induced by the balloon deflation that immediately preceded inflation (fig 3). In animal experiments it took at least two minutes for hyperkinesia to disappear after a minute of coronary artery ligation. ${ }^{13}$ Our study showed a slight decrease of the systolic thickening of the septum after the procedure (table 2 ). The proximal part of the septum is commonly the site of the most prominent hypertrophy in aortic stenosis. Reduction of systolic septal thickening soon after dilatation may show that hypertrophied myocardium is particularly susceptible to ischaemia and reperfusion and thus may be a manifestation of stunned myocardium. ${ }^{22}$ However, the technique used for. balloon dilatation of the aortic valve and the improvement of valve gradient and the clinical condition of the patients were similar to those reported in previous studies. ${ }^{1}$ Thus the minor reduction of systolic septal thickening can be regarded as clinically insignificant.

In our blind echocardiographic study of the left ventricular response to balloon dilatation of the aortic valve we found significant changes in left ventricular wall motion and wall thickness in relation to inflation and deflation of the balloon. The changes in left ventricular load undoubtedly contributed to the myocardial reactions but their character and time course resembled those seen in experimental and clinical ischaemia and reperfusion. Thus myocardial ischaemia and reperfusion may be important determinants of the left ventricular response to balloon dilatation of the aortic valve, and the potential impact on the myocardium of repeated ischaemia and reperfusion should be considered when percutaneous balloon dilatation of the aortic valve is performed.

1 Cribier A, Savin T, Berland J, et al. Percutaneous transluminal balloon valvuloplasty of adult aortic stenosis. Report of 92 cases. J Am Coll Cardiol 1987;9:381-6.

2 Isner JM, Salen DN, Desnoyers MR, et al. Treatment of calcific aortic stenosis by balloon valvuloplasty. $A m J$ Cardiol 1987;59:313-7.

3 McKay RG, Safian RD, Lock JE, et al. Assessment of left ventricular function after aortic balloon valvuloplasty in ventricular function after aortic balloon valvuloplasty in
adult patients with critical aortic stenosis. Circulation adult patients with

4 Pagani M, Vatner SF, Baig H, Braunwald E. Initial myocardial adjustments to brief periods of ischemia and reperfusion in the conscious dog. Circ Res 1978;43:83-92.

5 Distante A, Rovai D, Picano E, et al. Transient changes in left ventricular mechanics during attacks of Prinzmetal's angina: an M-mode echocardiographic study. Am Heart J 1984;107:465-74.

6 Alam M, Khaja F, Brymer J, Marzelli M, Goldstein S. Echocardiographic evaluation of left ventricular function during coronary artery angioplasty. Am J Cardiol 1986; 57:20-5.

7 Monaghan M, Thomas S, Jewitt D. Percutaneous aortic valvuloplasty for aortic stenosis [Abstract]. Circulation 1986;74 (suppl II):II-207.

8 Come PC, Riley MF, McKay RG, Safian R. Echocardiographic assessment of aortic valve in elderly patients with aortic stenosis and of changes in valve area after percutaneous balloon valvuloplasty. J Am Coll Cardiol percutaneous ball

9 Cyran SE, Kimball TR, Schwartz DC, Meyer RA, Steed D, Kaplan S. Evaluation of balloon aortic valvuloplasty with transesophageal echocardiography. Am Heart J 1988; 115:460-2.

10 Mortensen SA, Egeblad H. Endomyocardial biopsy guided by cross-sectional echocardiography. Br Heart J 1983; 50:246-51.

11 Suarez de Lezo J, Pan M, Romero M, Sancho M, Carrasco JL. Physiopathology of transient ventricular occlusion during balloon valvuloplasty for pulmonic or aortic stenosis. Am J Cardiol 1988;61:436-40.

12 Strandgaard $S$, Haunse $S$. Why does antihypertensive treatment prevent stroke but not myocardial infarction? Lancet 1987;ii:658-61.

13 Egeblad H, Haunsø S, Amtorp O. Regional dynamic behaviour of the canine myocardium following brief ischaemia: wall thickness, wall thickening and blood flow. Cardiovasc Res 1982;16:249-55.

14 Montague TJ, Johnstone DE, Spencer A, et al. Non-Q-wave acute myocardial infarction: body surface potential map acute myocardial infarction: body surface potential map and ventricul

15 Pandian NG, Skorton DJ, Collins SM, et al. Myocardial infarct size and threshold for two-dimensional echocardiographic detection: sensitivity of systolic wall thickening and endocardial motion abnormalities in small versus large infarcts. Am J Cardiol 1985;55:551-5.

16 Rousseau MF, Wyns W, Hammer F, Caucheteux D, Hue L, Pouleur $\mathrm{H}$. Changes in coronary blood flow and myocardial metabolism during aortic balloon valvuloplasty. Am J Cardiol 1988;61:1080-4.

17 Carroll RM, Laravuso RB, Schauble JF. Left ventricular function during aortic surgery. Arch Surg 1976;111: 740-3.

18 Egeblad H, Vilhelmsen R, Mortensen SA. Ischemic and postischemic ventricular wall motion abnormalities in Prinzmetal's angina provoked by hyperventilation. Am Heart J 1982;104:1105-7.

19 Gaasch WH, Bernard SA. The effect of acute changes in coronary blood flow on left ventricular end-diastolic thickness. Circulation 1977;56:593-8.

20 Goldberg S, Greenspon AJ, Urban PL, et al. Reperfusion arrhythmias: a marker of restoration of antegrade flow during ins: a marker of restoration of antegrade flow infarction. Am Heart $J$ 1983;105:26-32.

21 Roberts W. Am Heart $J$ 1983;105:26-32. valvulotomy. Am J Cardiol 1987;59:198-202.

22 Braunwald E. Kloner RA. The stunned myocardium: ploner RA. The stunned myocardium: prolonged, postischemic 